\title{
Perspectivas na avaliação em promoção da saúde: uma abordagem institucional
}

\author{
Perspectives in health promotion evaluation: \\ an institutional approach
}

José Ivo dos Santos Pedrosa 1

\footnotetext{
1 Departamento de Gestão da Educação na Saúde. Secretaria de Gestão do Trabalho e da Educação na Saúde.

Ministério da Saúde, Esplanada dos Ministérios, Bloco C, sala 717, 70058-900, Brasília DF. jose.pedrosa@saude.gov.br
}

Abstract The article intends to raise a few questions about perspectives in health promotion evaluation having as reference health public police in Brazil. It takes conceptual references from institutional analysis and it considers the health promotion evaluation like complex field having different concepts, methods and practices that are worked like analysators of this moment. It suggests that evaluation in health promotion must be a pedagogical dispositive of the meeting between the actors involved in the propose. It is basic for a participative construction of health promotion police.

Key words Health promotion, Evaluation, Institutional analysis, Evaluation in health promotion
Resumo O artigo se propõe a levantar algumas questões para o debate em torno das perspectivas na avaliação em promoção da saúde tendo como referência a política de saúde no Brasil. A partir de conceitos da análise institucional, considera a avaliação e a promoção da saúde campos complexos e polissêmicos com diferenças conceituais, metodológicas e práticas que servem de analisadores para a situação atual. Aponta para a avaliação como dispositivo de aprendizagem que emerge do encontro dos atores envolvidos com a proposta de mudança, fundamental para a construção participativa da política de promoção da saúde

Palavras-chave Promoção da saúde, Avaliação, Análise institucional, Avaliação em promoção da saúde 


\section{Introdução}

As perspectivas na avaliação de intervenções no campo da promoção da saúde encontram-se estreitamente relacionadas ao significado que se atribui à promoção da saúde e qual o sentido que se atribui à avaliação. Como todo campo em constituição, predomina uma polissemia de significados e de conceitos, assim como uma multiplicidade de ações que apontam para perspectivas de natureza diversa, desenhadas por atores de formação e filiação ideológica distintas.

A riqueza de elementos e a pluralidade de significações que se fazem presentes nos movimentos de constituição deste campo caracterizam-no como um espaço complexo, o que indica dificuldades em apontar perspectivas. Isto porque a avaliação, sendo da mesma natureza do objeto avaliado, é de grande complexidade.

A perspectiva da complexidade fortalece a idéia de que os problemas a serem considerados no campo são problemas não estruturados, que suscitam estudos voltados para o mapeamento dos elementos constitutivos de sua multiplicidade e suas articulações em cada contexto do movimento de saberes e práticas que constituem a promoção da saúde e sua avaliação.

Nestas condições, surge a necessidade de pensar em artifícios metodológicos que permitam recompor o objeto que se quer avaliar (as intervenções de promoção da saúde) na perspectiva de que os elementos que conformam e imprimem movimento ao campo e ao objeto expresso sob a forma de intervenções se tornem não apenas visíveis e contábeis, mas interpretados como dispositivos para a produção de referências para a avaliação.

Como diz Guattari (1987), a interpretação é o trabalho de situar a trajetória daqueles elementos para ver se eles têm condições de servir de indicadores de novos universos de referência, os quais podem adquirir uma consistência suficiente para provocar uma virada na situação.

Lapassade (1989) sugere a utilização de analisadores entendidos como dispositivos, que devem catalisar, aproximar e analisar o que até então estava disperso e dissimulado no conjunto da proposição. Estes analisadores, ao serem identificados e articulados, servem de referência para avaliar o que, como e quanto passou a ser instituído no cotidiano das pessoas, dos grupos, das organizações e instituições envolvidas.

Na visão de Baremblit (1994), um dispositivo se caracteriza porque o importante nele é seu funcionamento sempre simultâneo a sua formação e sempre a serviço da produção, do desejo, da vida, do novo.

Os movimentos dos campos em constituição se caracterizam pela dialética entre os elementos que passam a ser reconhecidos, identificados e instituídos no campo e as "pulsões" instituintes que forçam o alargamento de seus limites e a reconstrução de suas bases teóricas, metodológicas e práticas.

De maneira geral, poder-se-ia dizer que toda intervenção se caracteriza como dispositivo a partir dos efeitos não esperados, gerados tanto pelos recursos investidos que ficam incorporados à estrutura, quanto àqueles efeitos observados nos valores que governam o comportamento das pessoas e nos pressupostos do inconsciente que moldam a cultura organizacional (Fleury, 1992).

O entendimento de promoção da saúde tem sido ampliado, sobressaltando o papel protagonista dos determinantes gerais sobre as condições de saúde, compreendendo não somente as características dos indivíduos, mas as condições propiciadas pelo ambiente em seus aspectos físicos, sociais, econômicos e culturais.

Sob esta concepção, as ações de promoção da saúde incluem os indivíduos, suas famílias, as condições de trabalho, renda, alimentação, estilos de vida, lazer e ações que decorrem da formulação de políticas públicas que possibilitem aos indivíduos e comunidades a realização de escolhas em prol da saúde.

Além disso, promoção da saúde significa intervenções sustentadas pela articulação intersetorial e da participação social voltada para a consecução do direito à saúde, operando ações que visem à melhoria das condições de vida. Ações que podem ocorrer no espaço dos serviços de saúde, no espaço do parlamento e no espaço das comunidades.

A abrangência dos significados que a promoção apresenta e o sentido de mudanças expresso em suas proposições caracterizam-na como intervenção instituinte, pois opera no devir que serve de referência para o desenvolvimento de projetos de vida saudável. Assim, a avaliação das ações que ocorrem neste campo precisa responder antes à questão: o que, como e quanto das proposições da promoção da saúde foram instituídas sob a forma de políticas, programas, serviços e representações sociais.

Considerar a promoção da saúde sob o aspecto de intervenção instituinte de novas relações com as instituições, organizações, movi- 
mentos sociais e indivíduos, implica considerar o processo de institucionalização, resultado da relação dialética entre instituído e instituinte (Lourau, 1995), precondição para a avaliação. Identificar quais propostas políticas e técnicas que apostam em mudanças numa dada situação pode abranger instituições, organizações, grupos e pessoas, além das relações e das práticas de saúde.

A constituição do campo da promoção da saúde contempla pelo menos três níveis de institucionalização: os pressupostos e princípios teóricos orientadores de políticas nacionais de saúde, a presença de organizações e serviços produtores de ações organizadas em programas e projetos de promoção da saúde e ações promocionais que passam a fazer parte de atividades de grupos específicos da sociedade.

O núcleo de saberes que conforma a promoção da saúde tem como bases concepções oriundas da medicina social, da saúde pública e da saúde coletiva. A organização desses saberes se faz permitindo um movimento de ampliação dos espaços de atuação - famílias, grupos, comunidades e países; do objeto - instituições, organizações, atores sociais, indivíduos; e por aproximações com disciplinas como a sociologia, psicologia, epidemiologia e administração.

Os conceitos desenvolvidos transformamse em formulações que definem diretrizes discutidas e aprovadas em eventos internacionais, expressas em cartas e documentos que podem orientar políticas de saúde de âmbito nacional, estadual e local.

Articuladas a modelos assistenciais que se colocam como paralelos ou complementares aos modelos biologicistas de organização de serviços, as ações de promoção da saúde ganham características de programas, podendo reproduzir práticas decorrentes da impregnação de princípios da saúde pública clássica norteadas pelo mecanicismo da história natural das doenças. Podem também representar espaços de práticas participativas, construtivas e direcionadas para a autonomia dos indivíduos e das comunidades.

Ampliando sua abrangência para os espaços da sociedade, a promoção da saúde é desenvolvida por ações realizadas por diversos sujeitos sociais, sob várias modalidades de intervenção, envolvendo desde grupos específicos a movimentos sociais.

Neste sentido, avaliações no campo da promoção da saúde necessitam estar intimamente articuladas com o objeto avaliado pois é nesta relação que se constroem as questões, os critérios e os parâmetros para a avaliação. Isto significa dizer que, de maneira geral, a avaliação em promoção da saúde depende do nível de institucionalização pretendido que passa a representar o sucesso da aposta de mudança.

É necessário refletir sobre o processo de institucionalização da promoção da saúde, pois referências, instrumentos e metodologias para avaliação se encontram imersos nesse processo. Desvendá-los e articulá-los é um dispositivo de aprendizagem muito importante, porque acena para a possibilidade de uma avaliação mais inclusiva de questões, de formuladores de questões e de possibilidades de respostas.

\section{O processo de institucionalização da promoção da saúde}

A promoção da saúde, tal como é entendida pelo saber popular, tem aproximações com a visão holística e natural do processo de viver (do qual a saúde e a doença fazem parte) e que de certa forma prevalece nesse estado, como bandeira de luta social nos movimentos políticos até sua institucionalização como campo de práticas de saúde.

Agregada ao modelo preventivista, a promoção na forma de prática se define como intervenções que têm lugar em momentos anteriores à doença ou ao conhecimento sobre sua causa e prevenção: medidas inespecíficas que compreendem ações de baixa tecnologia realizadas por profissionais de formação média.

A educação em saúde torna-se a tecnologia apropriada para a promoção da saúde, na medida em que hábitos higiênicos adequados podem ser ensinados àqueles que não têm acesso às informações.

Este modo normal de pensar a trajetória da promoção da saúde sofre uma crise concomitante a outras crises que o mundo ocidental começa a vivenciar. Crise de Estados, de paradigmas, de valores, de saberes. A eclosão de movimentos sociais não necessariamente vinculados às lutas coorporativas, mas engendrados nas necessidades de grupos historicamente excluídos como negros, homossexuais, mulheres, meninos de rua e outros, serve de analisadora natural da situação estabelecida. Conseqüentemente, a promoção da saúde emerge como uma proposta transcendente ao setor saúde no sentido de influenciar mudanças nas políticas de saúde. 
Esta ressignificação da promoção da saúde, descolando-se do paradigma da história natural da doença, tem como referência o Relatório do Ministro de Saúde do Canadá, em 1974 - o Relatório Lalonde.

O relatório define como campos da saúde a biologia humana, o meio ambiente, estilos de vida e organização da atenção em saúde e a preocupação com o entorno ambiental (Brasil, 2002).

A promoção da saúde passa a ser considerada a estratégia para a consecução do modelo proposto que preconiza a regulação por parte do Estado, os investimentos em pesquisa, a eficiência dos serviços e a programação dos objetivos das intervenções.

Rootman e colaboradores (2001) consideram a publicação do Relatório Lalonde o momento inaugural da promoção da saúde como campo de saber e de intervenções. Em sua concepção, é o primeiro documento sobre promoção como política de governo. Consideram o documento um dispositivo disparador de ações em torno da política de promoção da saúde em vários países, indicando para a promoção duas dimensões: como conceito e estratégia, que podem ser usadas por governos, organizações, comunidades e indivíduos.

Este relatório certamente influencia, em 1977, a Assembléia Mundial de Saúde a lançar o slogan "Saúde para todos no ano 2000", baseado na extensão de cobertura dos serviços de atenção simplificada. Em 1978, em Alma Ata é realizada a I Conferência Internacional sobre Atenção Primária em Saúde, que reconhece a saúde como direito de todos e que seus determinantes são intersetoriais.

Torna-se perceptível que a ampliação do entendimento do fenômeno saúde/doença para além dos limites do campo estritamente sanitário, o reconhecimento do dever dos Estados de proporcionar os meios necessários para o bem-estar de seus cidadãos, o contexto dos países subdesenvolvidos e a crise mundial que serviram de cenário para as declarações de Alma Ata acenam para a introdução de novas modalidades de práticas de promoção e prevenção. E também para mudanças na concepção de educação em saúde, sob a influência de movimentos populares de saúde fundamentados pela pedagogia de Paulo Freire, que serão as bases para a educação popular e saúde (Vasconcelos, 2001).

Com o processo de democratização vivenciado na América Latina e particularmente no
Brasil, os movimentos políticos discutem a saúde como questão política, em discursos organizados em torno da discriminação, exclusão e precariedade do atendimento e da ineficiência das medidas de saúde pública.

Neste cenário, é possível dizer que para o Brasil o dispositivo/documento disparador foi a realização da VIII Conferência Nacional de Saúde e seu relatório, que definiu as diretrizes políticas da reforma sanitária brasileira. A temática dessa conferência - saúde é um direito de todos e dever do Estado - redireciona o objetivo e o processo de formulação de políticas públicas e contextualiza como dever do Estado a manutenção, promoção e proteção à saúde de seus cidadãos.

Colocando a saúde como direito, a reforma sanitária brasileira assume como campo de abrangência a situação de iniqüidade da saúde da população brasileira e toma como prioridade estratégica a organização de um sistema de atenção à saúde descentralizado, articulado, com gestão única em cada ente federado, acessível, resolutivo, de base local e com participação social.

A discussão da saúde como direito de cidadania reverbera nos movimentos políticos e se expressa na definição de saúde da Constituição Brasileira de 1988, que adota a Seguridade Social como modelo de proteção social integrando um conjunto de ações dos poderes públicos e da sociedade destinados a assegurar os direitos relativos à saúde, à previdência e à assistência social. O Estado brasileiro reconhece este desafio e legitima os esforços para alcançar a saúde como direito universal, instituindo um novo paradigma para a sua garantia, qual seja, a múltipla determinação do processo saúde/doença e a inter-relação da política de saúde com as políticas de outras áreas sociais e com as políticas econômicas (Brasil, Constituição Federal, 1988).

Além disso, a própria Constituição estabelece a participação da comunidade e o atendimento integral, com prioridade para as ações preventivas (sem prejuízo das atividades assistenciais), como diretrizes para a organização do sistema de saúde. A descentralização, com direção única em cada esfera de governo, completa o conjunto de diretrizes aprovadas pela sociedade, em acordo com a perspectiva da promoção.

Bodstein (2002) lembra os desafios metodológicos da avaliação de políticas públicas perante a contínua e permanente separação entre 
a formulação e a implementação dessas políticas. Conseqüências disso podem ser observadas na distância entre o discurso político institucionalizado e sua expressão operacional. Em países como o Brasil, o compromisso político assumido e explicitado em documentos oficiais demarca um território de possibilidades, mas não a efetivação da intenção em gesto.

Para a autora, programas e projetos de intervenção que, na prática, traduzem uma orientação e uma decisão política previamente tomada, não garantem sua execução e ou implantação, trazendo a indagação sobre em que medida uma determinada intervenção foi ou não de fato implantada (Bodstein, 2002).

Um outro nível do processo de institucionalização da política nacional de saúde se verifica no âmbito da organização do sistema orientada pelos princípios apontados acima, a disputa pela hegemonia entre modelos assistenciais orientadores do sistema único de saúde.

No mesmo cenário, mas com uma visão de outro ângulo, os princípios e propostas da promoção da saúde tornam-se evidentes nas justificativas e nos propósitos de vários programas que se revelam como estratégias para a implantação de políticas.

Nesse sentido, a promoção da saúde ganha objetividade em ações que se caracterizam como educativas, preventivas e de mobilização social, centradas na perspectiva dos agravos dos grupos vulneráveis, fazendo parte da maioria dos programas de saúde pública.

Mas, os níveis de institucionalização servem tão somente como marcadores para uma apropriação mais didática. Considerar o fato de as ações de promoção da saúde integrarem o elenco de ofertas nos programas de saúde, não significa a retração do movimento da promoção da saúde como diretriz direcionadora da política de saúde.

A legislação infraconstitucional - leis 8.080 e lei 8.142, de 1990 - não apenas reforça a concepção da promoção da saúde, como detalha a forma e mecanismos para a participação da comunidade na gestão do sistema e as condições para a promoção, proteção e recuperação da saúde no contexto da integralidade da atenção e da gestão descentralizada das ações e serviços (Brasil, 2003).

A Norma Operacional Básica do SUS 01/96 apresenta como bases para sua estruturação:

- Participação da população não somente nas instâncias formais, mas em outros espaços constituídos por atividades sistemáticas e per- manentes nos próprios serviços de atendimento, favorecendo a criação de vínculos entre o serviço e os usuários, caracterizando uma participação mais criativa e realizadora para as pessoas.

- As ações de saúde devem ser centradas na qualidade de vida das pessoas e de seu meio ambiente, bem como na relação da equipe de saúde com a comunidade, especialmente, com as famílias, impulsionando mudanças intersetoriais.

- O modelo epidemiológico considerado eixo de análise dos problemas de saúde, por entender que este modelo incorpora como objeto de ação as pessoas, o ambiente e os comportamentos interpessoais.

- Tecnologias em que os processos de educação e de comunicação social constituem parte essencial em qualquer nível de ação.

- A construção da ética coletiva que agrega as relações entre usuário, sistema e ambiente, e que possibilite modificações nos fatores determinantes, estimulando as pessoas a serem agentes de sua própria saúde.

- Demanda intervenções ambientais que suscitem articulações intersetoriais para promover, proteger e recuperar a saúde (Brasil, 2003).

Para consolidar o modelo proposto, a Norma Operacional institucionaliza o programa Saúde da Família e de Agentes Comunitários da Saúde como estratégias necessárias, definindo percentuais de financiamento como forma de incentivo aos gestores que aderirem à proposta de reorganização.

Ao definir os campos de atenção à saúde, a NOB/96 enfatiza a promoção como pertinente às responsabilidades do SUS, principalmente no que diz respeito às políticas externas ao setor saúde, que interferem nos determinantes sociais do processo saúde/doença das coletividades, como as políticas macroeconômicas relativas ao emprego, à habitação, à educação, ao lazer e à disponibilidade e qualidade dos alimentos.

Segundo o documento, as ações de política setorial em saúde, bem como as administrativas - planejamento, comando e controle - são inerentes e integrantes do contexto daquelas envolvidas na assistência e nas intervenções ambientais, explicitando ações de comunicação e educação em saúde como componentes da atenção à saúde.

Preconiza a NOB/96 a necessidade da implementação de mecanismos visando à integração das políticas e das ações de relevância para 
a saúde da população, como saneamento, recursos hídricos, habitação e meio ambiente.

A Norma Operacional de Assistência à Saúde (NOAS), editada pela portaria $n^{\circ} 95$ de 26/1/2001, considera e aprofunda as definições da norma anterior, enfatizando a regionalização e o aumento da eqüidade como necessários para aprofundar o processo de descentralização do SUS.

Esta mesma norma introduz a noção de territorialidade na identificação das prioridades de intervenção. Tal noção ultrapassa os limites físicos e se traduz na acessibilidade às ações e serviços que possam resolver os problemas de saúde do cidadão.

$\mathrm{Na}$ implantação do SUS, estratégias como a descentralização e a participação na sociedade no controle social do sistema foram acenadas como espaços de vivência para os princípios da promoção da saúde. Algumas ações e movimentos mais institucionalizados como o dos municípios saudáveis, escolas promotoras de saúde e outros, com apoio financeiro e técnico de organismos governamentais e não-governamentais foram sendo desenvolvidos, atingindo empresas e organizações voluntárias.

Programas e campanhas voltadas para vida saudável - atividade física, alimentação saudável, vigilância às doenças crônicas - representam importantes campos de intervenções para a promoção da saúde. A estas foram acrescidas as demandas da população vítima de violência e de condições de vida não adequadas.

No terceiro nível de institucionalização, a promoção da saúde é ressignificada como bandeira de luta dos movimentos sociais. A luta pela paz, contra a violência, o acesso à terra, projetos alternativos, enfim, a promoção da saúde na perspectiva de um devir instituinte.

Em síntese, esta discussão a respeito da promoção da saúde permite fazer um mapeamento da situação, para fins de análise:

- Os níveis de institucionalização são transitórios, dinâmicos e transversais ao processo de institucionalização de propostas direcionadas para provocar mudanças.

- Identificam-se os princípios da promoção da saúde institucionalizados nas políticas, nos modelos assistenciais e nos programas de saúde. - Os princípios da promoção da saúde são alinhados aos desejos da população, transformando-se em dispositivos para organizações em torno de projetos alternativos.

- A promoção da saúde se manifesta nos discursos políticos, na organização do sistema e como pauta de luta por parte dos movimentos sociais.

- Em algumas experiências de política local, os princípios da promoção da saúde direcionaram a política municipal e o desenho organizacional do sistema.

A promoção da saúde mostra-se transversal aos níveis de institucionalização tomados como analisadores e a partir desta reconstrução é possível colocar a discussão a respeito da avaliação. Como é possível avaliar o grau/nível de transversalidade? O que é transversal?

Para Guattari (1997), a transversalidade é uma dimensão que pretende superar os dois impasses, o de uma pura verticalidade e o de uma simples horizontalidade; ela tende a se realizar quando uma comunicação máxima se efetua nos diferentes niveis e, sobretudo, nos diferentes sentidos.

Numa perspectiva mais conservadora e tradicional, surgem questões a respeito da objetividade da transversalidade. Isso leva a um círculo no qual se retoma a questão inicial: como avaliar intervenções voltadas para mudanças que se mostram presentes nas políticas explicitadas, na legislação, na organização dos serviços, nos programas e nas ações cotidianas?

\section{A avaliação e alguns analisadores}

Os analisadores que podem ser identificados se encontram nas definições e concepções de avaliação, que vêm se acumulando nesse campo e o sentido que é possível atribuir à avaliação da promoção da saúde no atual estágio.

Considerando o caráter instituinte da promoção da saúde, ou seja, o sentido de mudança explícito em suas propostas de intervenção, as questões iniciais a respeito da avaliação remetem ao contexto no qual a intervenção é proposta; como a avaliação integra a proposta de intervenção; que informações são disponibilizadas; e a quem estas informações produzidas interessam, se aos gestores, usuários, financiadores e envolvidos.

De maneira geral, as avaliações também diferem quanto aos seus objetivos e podem ser definidas como:

a) avaliação de contexto - objetiva analisar a situação na qual a intervenção ocorre, incluindo a descrição dos elementos presentes nessa situação que representam importantes fatores de sucesso ou fracasso, tanto na entidade que vai receber a ação interventiva, na própria in- 
tervenção e no momento em que a intervenção é iniciada;

b) avaliação normativa - visa comparar o desenvolvimento da intervenção de acordo com regras estabelecidas anteriormente ou negociadas entre os participantes e envolvidos na intervenção;

c) avaliação estratégica - quando analisa a partir dos dados a respeito do contexto a coerência entre objetivos, metas e resultados alcançados, identificando as forças políticas interessadas e desenhando a viabilidade da intervenção;

d) avaliação de empoderamento (empowerment evaluation) - avaliação que se estabelece por meio da negociação entre avaliador e avaliado, também conhecida como avaliação comunicativa, objetivando acúmulo de poder por parte dos que desenvolvem a intervenção com as informações produzidas.

Tomando por base o desenvolvimento do campo da promoção da saúde e das avaliações suscitadas nesse processo, Rootman et al. (2001) identificam elementos nucleares para realizar uma certa cartografia dos conceitos, definições e práticas que tensionam estes campos determinando-lhes os movimentos.

Para os autores, a promoção é um campo multidisciplinar que contém indagações não resolvidas em termos de definições e práticas. Embora apresente divergências relativas aos objetivos, processos e ações, em linhas gerais, segundo os autores, a promoção da saúde parece ter consenso em torno da concepção de estratégia para promover o bem-estar individual e coletivo.

Analisando o estado da arte da avaliação em promoção da saúde, os autores identificam posições diversas em relação à avaliação de programas, ao processo de julgar (valoração), à construção e à utilização do conhecimento e às práticas avaliativas que têm sido realizadas.

Quando a promoção da saúde se caracteriza como um programa, definido como artefatos humanos compostos de recursos articulados (disponíveis) para criar serviços e atividades direcionadas a um problema em dado contexto, a uma determinada população, que se espera produza efeitos na situação, emergem posições que priorizam a avaliação do processo, focalizando recursos, atividades e posições que avaliam os resultados trazidos pela intervenção nas relações entre atividades, serviços e mudanças na situação.

Acrescenta-se à discussão a análise do contexto como parte da avaliação por definir e si- tuar o problema a ser enfrentado e a busca de teoria do programa que justifique sua relação com o contexto (Hartz, 1999).

Em termos dos valores éticos agregados às intervenções em promoção da saúde, Rootmam et al. (2001) identificam diferenças quando valores como justiça social são colocados de maneira prescritiva às intervenções e quando, ao contrário, são valores construídos com os interlocutores e envolvidos que modelam a intervenção.

A avaliação como atividade resultante e propiciadora da construção de conhecimentos compreende desde posturas positivistas centradas na idéia de causalidade detectada em estudos de desenhos experimentais que sobrepõem a validade interna a posições construtivistas em que a validade do conhecimento produzido encontra-se no compartilhamento de saberes dos envolvidos, compreendendo ainda posições intermediárias que sugerem a adaptação do conhecimento produzido pela avaliação da natureza da questão que pretende responder.

No que diz respeito à utilização do conhecimento produzido pela avaliação, as divergências aparecem quanto ao sentido instrumental ou finalístico que assume no processo avaliativo e na relação com os envolvidos. E, finalmente, os autores apontam diferenças observadas nas práticas avaliativas referentes ao papel do avaliador, às questões que orientam a avaliação, ao desenho da investigação avaliativa e às atividades concernentes.

Este conjunto de questões que aqui são consideradas analisadores da avaliação em promoção da saúde necessita ser rearticulado para ganhar o sentido de referência para a avaliação a ser desenvolvida, considerando-se que avaliar implica o ato de valorar algo em relação a um padrão/referência tomado como possível.

\section{Os analisadores como referência: perspectivas de avaliação em promoção da saúde}

Dos questionamentos feitos até aqui alguns analisadores podem servir de base para a discussão mais prospectiva. $\mathrm{O}$ primeiro diz respeito à formulação da proposta de promoção da saúde.

Nesse sentido, há que reconhecer qual o lugar que a proposta ocupa em sua relação com a política de saúde e a organização do sistema de saúde. Necessário se faz identificar qual a dire- 
cionalidade da proposta no contexto em que surge e pretende intervir e o ambiente (lócus) no qual a proposta circula: na política, nos serviços, nos programas e nas ações promocionais.

Este cuidado preliminar se justifica diante da possibilidade da proposta apresentar-se superdimensionada, expressando produtos e resultados que não poderão ser alcançados utilizando processos que não se coadunam com as expectativas. Em casos extremos, a avaliação é inviabilizada pela incapacidade de gerar a pergunta central da avaliação.

Uma estratégia metodológica para fazer frente ao problema é identificar as possíveis ambigüidades que a proposta pode conter. As ambigüidades podem se tornar visíveis no momento em que a proposta de promoção da saúde é explicitada, no lugar que ocupam seus porta-vozes e nas possíveis adesões que pode suscitar. Além disso, é possível perceber incoerências da proposta diante da cultura instituída no espaço de intervenção.

Considerando os analisadores perceptíveis nas diferentes concepções de promoção da saúde, observa-se que o arcabouço conceitual da promoção da saúde permite, para fins de análise, a construção de três grandes matrizes: a promoção da saúde atrelada à concepção de risco epidemiológico e social, a promoção da saúde articulada a propostas mais amplas de desenvolvimento sustentado e uma terceira matriz, intermediária, voltada para ações de intersetorialidade, participação ativa da população com prioridade para o desenvolvimento local.

Na primeira matriz existe uma tendência a estruturar os problemas de saúde, a partir da concepção de risco e vulnerabilidade por meio de critérios objetivos, o que obviamente define com "precisão" o problema, os objetos, a intervenção, os resultados esperados. Neste sentido, indivíduos ou grupos têm o lugar de unidade amostral/unidade de intervenção, a subjetividade e a dinâmica da vida são subsumidos a variáveis epidemiológicas. Para Hartz (1999), avaliar um programa é muito mais que apenas estimar os diferenciais de risco ao final de uma intervenção.

$\mathrm{Na}$ segunda matriz na qual se agrupam as propostas de intervenção de caráter mais amplo, presentes nos pressupostos e articuladas aos discursos que orientam as políticas de saúde, as ações desenvolvidas ocorrem no nível de macropolíticas, em algumas vezes sem nenhuma tradução no nível das práticas organizacionais.
Neste sentido e tomando o Sistema Único de Saúde brasileiro como referência, avaliar aspectos estreitamente vinculados ao ideário da promoção da saúde como a descentralização político-gerencial do sistema e a participação da população, implica qualificar e institucionalizar a avaliação nos diversos níveis de governo comprometidos com as mudanças do modelo assistencial do SUS (Hartz, 2002).

Silva (1999), discutindo a avaliação do processo de descentralização da política de saúde brasileira, considera como objeto a municipalização da saúde, aponta as perspectivas metodológicas integradoras que articulam aspectos quantitativos e qualitativos disponíveis no repertório avaliativo. Sugere dois atributos para o monitoramento do processo de descentralização - a cobertura e a efetividade -, considerando que monitorar significa promover uma avaliação sistemática sobre algumas características das práticas ou objetos.

Mais difícil se torna a tarefa de avaliar outro eixo organizacional do SUS, a integralidade da atenção. Para Cecílio (2001), o modo concreto de articular ações dizendo-as integrais no cuidado define o patamar ético e de programação e avaliação da qualidade da assistência, dimensões situadas no núcleo duro do planejamento e gestão em saúde.

Para Hartz (2002), institucionalizar a avaliação exige a definição de uma política para avaliar políticas que apresente um mínimo de diretrizes relativas aos propósitos e recursos atribuídos à avaliação. Estão entre estas diretrizes a estrutura: a localização e abordagens metodológicas das instâncias de avaliação; a prática: as relações estabelecidas com a gestão; e a tomada de decisões - utilização.

Se as diretrizes mínimas apontadas acima se mostram imprescindíveis para a institucionalização da avaliação como política, ter a avaliação instituída na estrutura da política requer alguma cautela em razão das inúmeras transformações que as intenções, diretrizes e normas que orientam a política sofrem ao serem implantadas no âmbito federal, estadual e municipal de gestão do sistema.

Este cuidado se torna particularmente importante no Brasil, diante da difícil e desigual realidade socioeconômica, da extensão territorial nacional, da heterogeneidade dos problemas regionais, das distintas capacidades locais e da diversidade cultural. É preciso promover práticas solidárias, criativas e flexíveis que contribuam para superar relações excludentes e si- 
tuações de iniqüidade, e concretizem propósitos sociais de bem-estar para todos.

Nesse sentido, a questão que se levanta é a respeito do instituído ser capaz de manter sua potência instituinte, ou seja, como equacionar a necessidade de a avaliação ser desenhada em função da plasticidade e flexibilidade do objeto e ao mesmo tempo apresentar os critérios de utilidade, factibilidade, propriedade e acurácia (Pedrosa, 2001). Isto, considerando-se que promover a saúde dos indivíduos e de suas comunidades significa atuar sobre os determinantes da saúde e da doença que se encontram na dimensão subjetiva de cada indivíduo, na objetividade com que a vida se faz presente para pessoas e comunidades e nos processos sociais que se expressam na dimensão política e cultural.

A terceira matriz diz respeito a programas e projetos que não se limitam à esfera do setor saúde, têm abrangência localizada em territórios definidos, definem grupos populacionais como sujeitos da intervenção e procuram articular-se com outros setores sociais.

Bryant (2002) considera que o desenvolvimento de políticas públicas saudáveis e de programas locais de saúde pública tem sido fundamentado por conhecimentos científicos estreitamente relacionados aos modelos clínico e epidemiológico. Segundo o autor, esta centralidade tem levado, por parte de autores norteamericanos, a uma ênfase na temática sobre estilos de vida como um problema que modela os programas de atenção à saúde e à utilização de métodos quantitativos para avaliar os efeitos de intervenções dessa natureza. Contrariamente, tem deixado de priorizar as questões sociais e econômicas que exercem influência na saúde e no bem-estar, como a pobreza e o ambiente.

Considerando a necessidade de identificar propostas de avaliação mais condizentes com a idéia da totalidade da saúde, não somente delimitada pelo arsenal de conhecimentos e tecnologias existentes no campo médico, mas abarcando as propostas de desinstitucionalização da atenção à saúde, Furtado (2001) apresenta uma proposta de método construtivista. Tratase de uma avaliação participativa - método no qual articula os preceitos da avaliação de quarta geração ou avaliação de empoderamento sugerido por Guba \& Lincoln (1989) com o Método da Roda sugerido por Campos (2000).

O Método da Roda, grupo de pessoas em círculo debatendo determinada questão, se apresenta como um dispositivo para pensare transformar o Estado, as relações econômicas e sociais, mas também as relações familiares, entre os gêneros, os sistemas de saúde, de educação, as igrejas, partidos, movimentos, alterar, enfim, o modo de gestão das instituições. Tudo passível de críticas e mudanças. Uma ampliação do espaço tradicional da politica e, ao mesmo tempo, uma reconstrução dos modos de fazer política (Campos, 2000).

Para Furtado (2001), a articulação dos dois métodos contribuiria para a construção de novos sentidos e significados da intervenção e da avaliação, considerando dimensões como o estabelecimento de contratos e compromissos, negociações concretas entre desejos e interesses, mediados por uma postura ética que garante igual valor às manifestações dos implicados na Roda. Esse processo define o problema, a intervenção e a avaliação.

Assim resgata-se o papel da avaliação como dispositivo pedagógico para grupos de implicados na intervenção, que no dizer de Silva \& Brandão (2003) significa a elaboração, negociação e aplicação de critérios explícitos de análise, em um exercício metodológico cuidadoso e preciso, com vistas a conhecer, medir, determinar ou julgar o conteúdo, mérito, valor ou estado de um determinado objeto, a fim de estimular e facilitar processos de aprendizagem e de desenvolvimento de pessoas e organizações.

$\mathrm{Na}$ conjuntura atual é possível formular políticas públicas de saúde com a participação da sociedade, nas quais os projetos de vida para a autonomia, construídos pela sociedade, sejam políticas do Estado Democrático Popular. Sendo assim, é necessário que a sociedade civil e os movimentos sociais se tornem formuladores dessas políticas, participem de sua gestão e exerçam o controle público de sua implementação.

Quando as relações entre movimentos sociais e Governo se caracterizam como relações em que o primeiro exerce o papel do sujeito vitimado, de apresentador de demandas por recursos e o segundo representa o doador destes recursos, institui-se uma relação na qual não existe comunicação, nem condições para que seja produzido um acontecimento.

Assim, no processo de institucionalização das ações de promoção da saúde e da avaliação dessas intervenções é possível trabalhar na micropolitica do encontro com a sociedade civil considerada interlocutora. O encontro é espaço de aprendizagem no qual as ações de promoção da saúde podem ser concebidas como dispositi- 
vos instituintes de novas relações entre pessoas, saberes e práticas ampliadas de saúde. Do encontro entre estes atores é possível construir projetos voltados para a promoção da saúde.

A avaliação, como dispositivo pedagógico na construção de políticas de promoção da saúde, assume também o caráter de instrumento de responsabilização de cada ator nessa construção, que define seu lugar no processo e seu projeto a partir da reflexão a respeito do como e para que avaliar. Sendo necessariamente participativa, a avaliação em promoção da saúde pressupõe negociação, pactuação e decisão coletiva entre os atores envolvidos, organizada em co-gestão, conduzidas pelos diferentes atores de uma instituição, organização ou movimento social com a intencionalidade de transformação.

Como campo complexo e ação especializada, a avaliação em promoção da saúde não ne- ga os critérios de competência científica observados nas pesquisas avaliativas. Ultrapassa, entretanto, a racionalidade instrumental na medida em que é orientada mais pelos princípios da comunidade do que do mercado ou do Estado, trabalhando na dialética entre a regulação (o instituído) e a emancipação (instituinte), sendo, portanto, transdisciplinar e multicultural.

Finalmente a avaliação em promoção da saúde é uma ação permanente. Neste sentido deve ser descentralizada radicalmente, ascendente, sendo suas indagações originadas no espaço da própria intervenção em seu contexto. Ou seja, de dentro para fora, de modo que contribua para a democratização institucional, o desenvolvimento da capacidade de aprendizagem política e de enfrentamento criativo dos problemas que fazem parte da vida - a maior expressão da promoção da saúde.

\section{Referências bibliográficas}

Baremblit G 1994. Compêndio de análise institucional e outras correntes: teoria e prática. (2a ed.). Rosa dos Tempos, Rio de Janeiro.

Bodstein R 2002. Atenção Básica na Agenda da Saúde. Ciência \& Saúde Coletiva 7(3):401-412, 2002.

Brasil. Ministério da Saúde 2002. Secretaria de Políticas de Saúde. Projeto de Promoção da Saúde. As cartas de promoção da saúde. Ministério da Saúde, Brasília.

Brasil. Conselho Nacional de Secretários de Saúde 2003. Legislação do SUS. CONASS, Brasília.

Bryant T 2002. Role of knowledge in public health and health promotion policy changes. Health Promotion International 17(1):89-98.

Campos GW S 2000. Um método para análise e co-gestão de coletivos. Hucitec, São Paulo.

Cecílio LCO 2001. As necessidades de saúde como conceito estruturante na luta pela integralidade e eqüidade na atenção em saúde. In R Pinheiro \& R Mattos. Os sentidos da integralidade na atenção e no cuidado à saúde. IMS-UERJ-Abrasco. Rio de Janeiro.

Fleury MTL 1992. O desvendar a cultura de uma organização: uma discussão metodológica, pp.15-27. In MTL Fleury \& RM Fischer (orgs.). Cultura e poder nas organizações complexas. Atlas, São Paulo.

Furtado JP 2001. Um método construtivista para a avaliação em Saúde. Ciência \& Saúde Coletiva 6(1):165-181.

Guattari F 1987). Revolução molecular: pulsações políticas do desejo. (3a ed.). Brasiliense. São Paulo.

Guba EG \& Lincoln YS 1989. Fourth generation evaluation. Sage Publications. Thousand Oaks.

Hartz ZMA 1999. Avaliação dos programas de saúde: perspectivas teórico-metodológicas e políticas institucionais. Ciência \& Saúde Coletiva 4(2):341-353.
Hartz ZMA 2002. Institucionalizar e qualificar a avaliação: outros desafios para a atenção básica. Ciência \& Saúde Coletiva 7(3):401-412.

Lapassade G 1989. Grupos, organizações e instituições. (3a ed.). Francisco Alves, Rio de Janeiro.

Lourau R 1995. Análise institucional. (2a ed.). Vozes, Rio de Janeiro.

Pedrosa JIS 2001. Avaliação de práticas educativas em Saúde. In E Vasconcelos (org.). A palavra na saúde e nos gestos. Hucitec, São Paulo.

Rootman I, Goodstadt M, Potvin L, Springett J. 2001. A framework for health promotion evaluation. Evaluation in health promotion: principles and perspectives. Who Regional Publication European Services 92:7-38.

Silva LMV 1999. Avaliação do processo de descentralização das ações de saúde. Ciência \& Saúde Coletiva 4(2):331-339.

Silva RR \& Brandão D 2003. Os quatro elementos da avaliação. Olho Mágico-Boletim da Rede Unida 10(2):59-66.

Vasconcelos EM 2001. Redefinindo as práticas de saúde a partir de experiências de Educação Popular nos serviços de saúde. Interface - Comunicação, Saúde, Educação 5(8):121-126.

Artigo apresentado em 26/4/2004

Aprovado em 6/6/2004

Versão final apresentada em 26/6/2004 